\title{
CFD Analysis and Optimization of Geometrical Modifications of Ahmed Body
}

\author{
Sagar Sharma ${ }^{1}$, Saurabh Banga ${ }^{2}$, Rohit Singh Dungriyal ${ }^{3}$, \\ Mohammad Zunaid* ${ }^{4}$, Naushad Ahmad Ansari ${ }^{5}$, Suresh Lal ${ }^{6}$ \\ ${ }^{1,2,3}$ U.G Student, Department of Mechanical \& Automobile Engineering, Delhi Technological University, \\ Bawana Road, Delhi, India \\ 4, 5 Assistant Professor, Department of Mechanical Engineering, Delhi Technological University, Bawana Road, \\ Delhi, India \\ ${ }^{6}$ Dy. Director, CEFEES (DRDO), Delhi, India.
}

\begin{abstract}
This project is modeled around reducing the overall drag and lift coefficient by modifying the geometry and comprehending the underlying rootphenomenon for the variation in these drag values. The designing of models used in this project is done through SOLIDWORKS, and CFD simulation in FLUENT (ANSYS) respectively. The purpose of this project is to reduce the inefficiency caused due to aerodynamic factors of drag, lift by presenting development, design and optimization of the geometry modifications of the base model. The improvement of aerodynamic behaviour around the periphery of the model is investigated through numerical simulation. The Ahmed Reference Body having 10 degree rear slant angle has been taken as benchmark model in this paper. Simulation of variants of multifarious modifications and examination of their results is performed to find the best suited variant form (dimension) to boost the aerodynamic efficiency of the model. CFD simulations on Ahmed Body were carried out in order to serve as a benchmark for validating all the simulation results. The steady-state simulations are based on the Reynolds averaged Navier-Stokes equations, with turbulence closure provided through two-equation k-epsilon realizable models.
\end{abstract}

Keywords: Ahmed body, CFD, drag, k-Epsilon realizable model, lift.

\section{Introduction}

As the burning of fossil fuels is becoming a more pressing unsustainable issue in lieu of the environmental degradation and increasing energy prices, automobile manufacturers are introducing more fuel efficient cars to the market. One of the main contributors to fuel consumption is the aerodynamic drag since it accounts for more than half of the engine power at highway speeds.

The pressure drag which highly depends on vehicle geometry is because of boundary layer separation from rear trailing end and consequent formation of wake region behind the vehicle. The location of separation determines the size of wake region and consequently it determines the value of aerodynamic drag coefficient.

The drag coefficient quantifies the resistance of an object in a fluid environment. The lower the drag coefficient, the more efficient the car is. The drag coefficient also affects the handling in addition to affecting the top speed of a vehicle. Cars possessing low drag coefficients are desirable, but decreasing the drag drastically can reduce the downforce (increase lift) and lead to loss in road traction and increase in chance of accidents. There are numerous ways to modify a car to optimize its aerodynamics by decreasing the drag coefficient. To streamline the exterior, certain aftermarket accessories such as the roof rack, mud flaps, spoilers, and radio antenna can be removed. Wheel covers, a partial grille block, a properly designed under tray, fender skirts, and a modified front bumper can be utilized to reduce the drag coefficient.

The drag reducing methods are classified into active or passive control methods based upon whether these methods consume energy to control the flow of air or not: Passive flow control strategies such as vortex generators are accompanied by vehicle geometry modifications. Whereas, active control is performed by using actuators that require power, generally extracted from the prime mover of the vehicle. The visible part of these systems includes mobile walls, circular holes or slots distributed over the vehicle surface where the flow must be controlled. Active control methods such as suction, blowing seem constrained to theory and they may not be practical or feasible. In addition, it is yet to be determined how to optimize their control performances. The Ahmed body is a bluff body whose shape is simple enough to model, while maintaining certain important carlike geometry features.The Ahmed body was first proposed by Ahmed(1984) [1]. To reduce the computational cost, a symmetry plane is introduced to model half of the model.

For the model, the flow is turbulent, which is based on the Reynolds number determined by the body length and inlet velocity. The simulation solves for the turbulent kinetic energy and dissipation energy in addition to the velocity and pressure fields. 


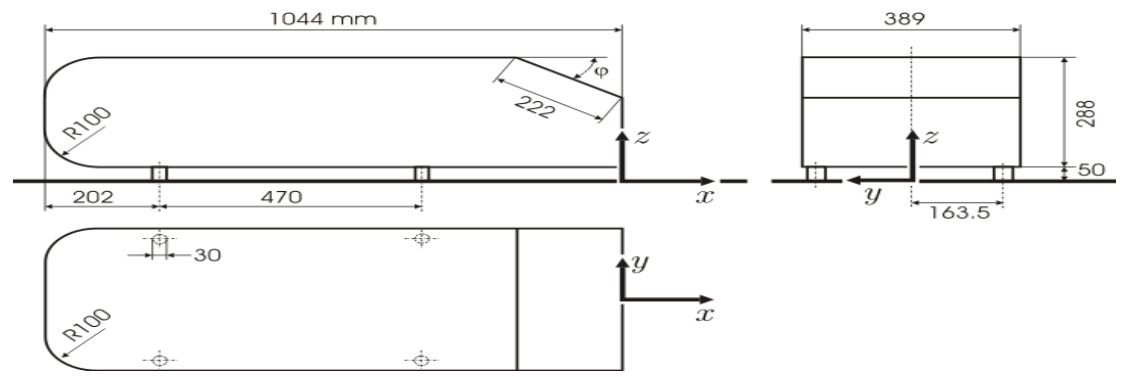

Fig. 1: Geometry of Ahmed model

Manipulation of the rear slant angle of the generic Ahmed body reference model was carried out previously [2], to determine the optimum angle configuration for the least drag coefficient with velocity of air at inlet being $40 \mathrm{~m} / \mathrm{s}$. The drag coefficient and the corresponding lift coefficient were plotted.The pressure distribution and velocity contours for the various rear slant angles were also investigated to comprehend the aerodynamic behavior around the model.

The Ahmed Body with 10 degree rear slant angle has been chosen as the benchmark model for carrying out computations for studying of the aerodynamic parameters. This paper has the following objectives:

1. To investigate and comprehend the variation of drag and lift coefficient by conducting numerical simulation on the benchmark modeland numerous configurations of 6multifariousgeometrically modified models of the benchmark body. The various configurations are obtained by varying the associated governing geometrical parameter for each modified model.

2. To exhibit the velocity contours foroptimumconfiguration of each geometrically modified model, focusing at the wake region of the body.

3. To portray pressure distribution over the entire body of optimum configuration for a particular geometrical modification of the benchmark model.

\section{Literature Review}

Han et al. (1992) [3] performed aerodynamic shape optimization on Ahmed body with three shape parameters: backlight angle $\left(0^{\circ}\right.$ to $\left.30^{\circ}\right)$, boat tail angle $\left(0^{\circ}\right.$ to $\left.30^{\circ}\right)$ and ramp angle $\left(0^{\circ}\right.$ to $\left.20^{\circ}\right)$. The $\mathrm{k}-\varepsilon$ turbulence model CFD solver was coupled to an optimization routine. In this study, an analytic approximation function of the objective function (drag coefficient values from CFD analysis) was created in terms of the design variables. The optimization was then performed on this approximation function and optimum parameters were found. The CFD analysis was again performed with this optimum set of parameters and the objective function was updated with new results. This process was continued until the parameters for minimum drag were obtained. The optimization process revealed that the optimum rear body parameters are backlight angle of $17.8^{0}$; boat-tail angle of $18.9^{\circ}$; and ramp angle of $9.2^{\circ}$.

Pujals et al. (2001) [4] experimentally investigated the potential of separation control strategy based on large-scale coherent streaks. These streaks are generated by means of an array of cylindrical roughness elements on a highly three-dimensional flow subject to an adverse pressure gradient due to geometrical constraints. The geometry chosen was Ahmed body with $25^{0}$ rear slant at Reynolds number $\operatorname{Re}_{\mathrm{L}}=1.35 \mathrm{X} 10^{6}$. Various configurations of span-wise spacing and stream-wise locations on the roof were investigated and substantial drag reduction was obtained for all sets of wavelength and stream-wise locations. The overall maximum total drag reduction measured was about $10 \%$. PIV as well as static pressure measurements on the slanted surface prove that the drag reduction is due to the suppression to the re-circulation bubble.

Englar and Robert (2001) [5] at Georgia Tech implemented pneumatic devices on heavy duty vehicles and conducted wind-tunnel investigations on a generic Heavy Vehicle model with blowing slots on both the leading and trailing edges of the trailer. The trailing edge was provided with a fixed curved surface and a tangential slot ejecting a jet sheet which remained attached to the curved surface by a balance between subambient static pressure on the surface and centrifugal force. Drag coefficient reductions of as much as $50 \%$ were produced by blowing with internal pressures of only 1.0 psig; $C_{D}$ values as low as 0.13 (an $84 \%$ drag reduction from the baseline Heavy Vehicle model) were measured at increased blowing rates.

Zunaid et al. (2014) [6] designed and implemented the curved boat tail configuration without the AFC device on a generic SUV design. The working principle of the add-on was based on the Coanda effect, causing the airflow over and under the SUV to take a curved path resulting in blowing of air in the immediate wake region. They investigated its effect on various aerodynamic parameters reporting an increase in the average pressure in wake region along with a significant drag reduction of $8.013 \%$. 
Lamond et al. (2009) [7] conducted a preliminary investigation into the use of base bleed on a production SUV using CFD analysis.For the geometry studied, a bleed outlet applied to the upper portion was found to be most effective in reducing drag but there may be difficulty in implementation as bleeding air from a high pressure region towards the top would be difficult. It was found that bleed through the sides of the vehicle was also effective and this would allow venting from the sides, underside or roof The paper portrayed that the reduction in drag increased as the mass flow rate of air increased, when the flow was deflected at the outlet; a mass flow rate of under $1.5 \mathrm{~kg} / \mathrm{s}$ could reduce the drag coefficient by $10.7 \%$ for an outlet on the upper section of the rear of the vehicle.

Bahram et al. (2001) [8] experimented with a square back bluff body using tail and boat plate like addon for drag reduction. The mean pressure results showed a significant increase in the base pressure with the drag reduction device and it also suppresses large-scale turbulent motions in the wake. The results also showed a reduction of the turbulence intensity as well as a rapid upward deflection of the underbody flow with the device in place and confirmed that the separated flow from the trailing edge of the model reattached on the extended plate (add-on device) which is similar to that of a boat-tail effect using the concept of Coanda effect [9] for drag reduction by up to $20 \%$.

Al-Marzooqi et al. (2012) performed an experiment on Ahmed body to improve the aerodynamic efficiency of the SUV model which he used in his paper [10]. The add-on device in the form of lateral guide vanes were installed at the rear end of the SUV to direct the air flow into the low pressure wake region at the rear of the vehicle. This would improve the pressure recovery in the wake due to mass addition of the directed air, which in turn would reduce form drag and hence the overall drag of the box. The chord length of the guide vanes was taken as L/8, where L represents the length of the car box. The angle of attack of the lateral guide vanes was also subjected to various manipulations of angles and consequently, significant reduction in the drag coefficient up to $17 \%$ was noted.

Gustavsson and Melin (2006) [11] at KTH performed the experiments to find the advantages of the VGs when applied to blunt bodies. Wind tunnel was used to perform the simulation of the bluntbody with the VG located at the rear slant angle, behind the shoulder of the body. The dimensions of the main body were given as $\mathrm{L}=0.95 \mathrm{~m}, \mathrm{~W}=0.50 \mathrm{~m}$ and $\mathrm{H}=0.30 \mathrm{~m}$, with rear slant angle of 40 degree. There were two wind tunnel tests conducted by them. In the first test, the main objective was to determine the effectiveness of VGs at redirecting airflow which wassuccessfully confirmed after the test. The second wind tunnel test was conducted with the VGs placed before the rear slant angles in conjunction with a combination of boat tail angles of 20, 40, 60 and 90 degrees and different VG height and length were tested. The least drag coefficient of 0.4540 was obtained in the results.

\subsection{Benchmark Model}

\section{Geometry Modifications Of Ahmed Body}

The Ahmed body represents a simplified, ground vehicle geometry of a bluff body type. Its shape is simple enough to allow for accurate flow simulation but retains some important practical features relevant to automobile bodies. Ahmed body with the rear slant angle of 10 degree (as shown in fig-2) is selected as the benchmark model. Different modifications are carried out on this geometry of Ahmed body to obtain best suited configuration for minimum drag coefficient.

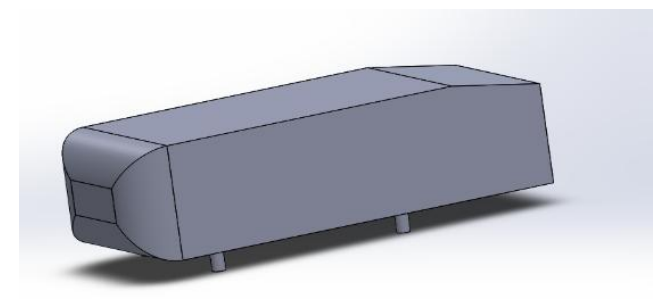

Fig. 2 - Ahmed body with rear slant angle 10 degree

\subsection{Roof Panel Cambering}

Roof cambering (as shown in fig-3) reduces drag due to air flowing past thesmooth curvature at the top of Ahmed body resulting in increased turbulence and thus, delayed boundary layer separation. Roof camber should not be increased at the expense of enlarging the frontal area as this would be counter-productive and would increase drag coefficient. 


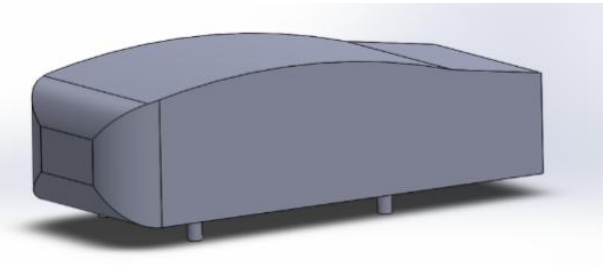

Fig.3 - Roof panel cambering

\subsection{Side Panel Cambering}

Side panel cambering (as shown in fig-4) results indrag reduction as a consequence of air flowing past the smoothcurvature at the side of Ahmed body, reducing the formation of vortices due to increased turbulence and thus, delayed boundary layer separation. As in case of roof camber, side camber also should not be increased at the expense of enlarging the frontal area as this would be counter-productive and would increase drag coefficient.

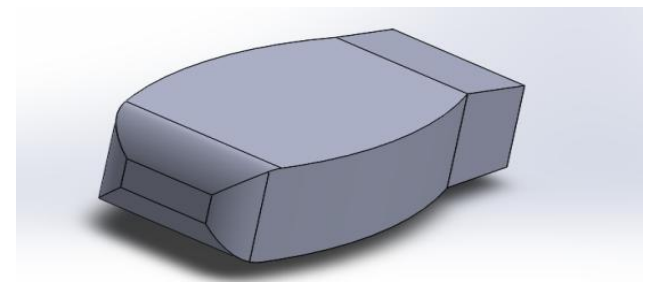

Fig.4- Side panel cambering

\subsection{Underbody Rear End Upward Taper}

Tilting the underbody rear end upwards (as shown in fig-5) produces a diffuser-like effect which gives a promising scope in reducing drag coefficient. However, it is important to select an optimum angle and ratio of length of taper for best results. Length of taper is taken same as upper body slant length i.e. $222 \mathrm{~mm}$.

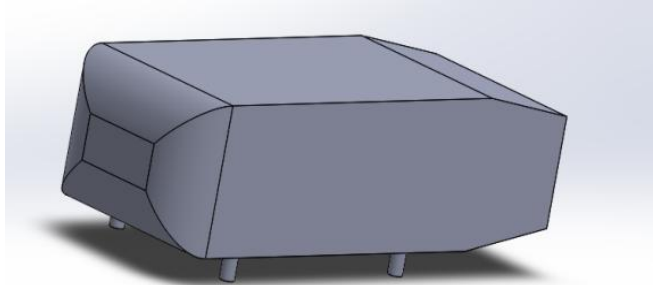

Fig.5- Underbody rear end upward taper

\subsection{Rear Side Panel Taper}

Tapering the rear side panel inwards (as shown in fig-6) reduces the drag coefficient due to reduced wake area at the rear. Taper is done on both sides equally with different magnitudes.

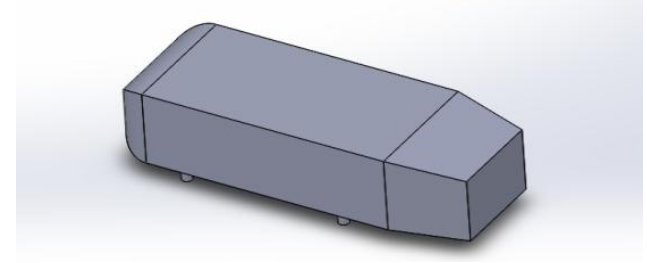

Fig.6- Rear side panel taper 


\subsection{Rear End Tail Extension}

Reduced drag coefficient is obtained with increment in extension of the rear tail (as shown in fig-7) but this shape is inconvenient to accommodate from the perspective ofpractical application and aesthetic design considerations. Conversely, if the rear end tail is cropped at various lengths and curved downwards there is an increase in drag coefficient with each reduction in tail length beyond the rear wheels.

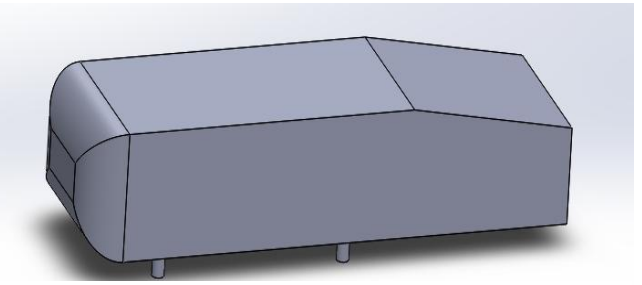

Fig.7- Rear end tail extension

\subsection{Chamfered Nose}

In this model, instead of using fillet on nose ofthe frontal surface of the Ahmed body, chamfering is done to $100 \mathrm{~mm}$ (as shown in fig-8). However due to the sharp edges, boundary layer separation occurs and consequently drag coefficient increases.

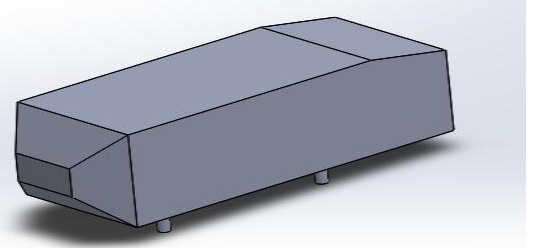

Fig.8- Frontal surface chamfered

\section{Numerical Simulation}

The ANSYS Workbench platform is the framework upon which the industry's broadest and deepest suite of advanced engineering simulation technology is built. An innovative project schematic view ties together the entire simulation process, guiding the user through even complex multi-physics analysis with drag-and-drop simplicity. Firstly, Solid works file is attached into ANSYS Workbench. Next, an enclosure is created in which is air surrounding around body having dimensions $5 \mathrm{~m}$ from front and $7.5 \mathrm{~m}$ from rear in the 'Design Modeller' in ANSYS. Then, the body is subtracted from air to create single body domain. This subtracted body represent Ahmed body walls around the surrounding air.

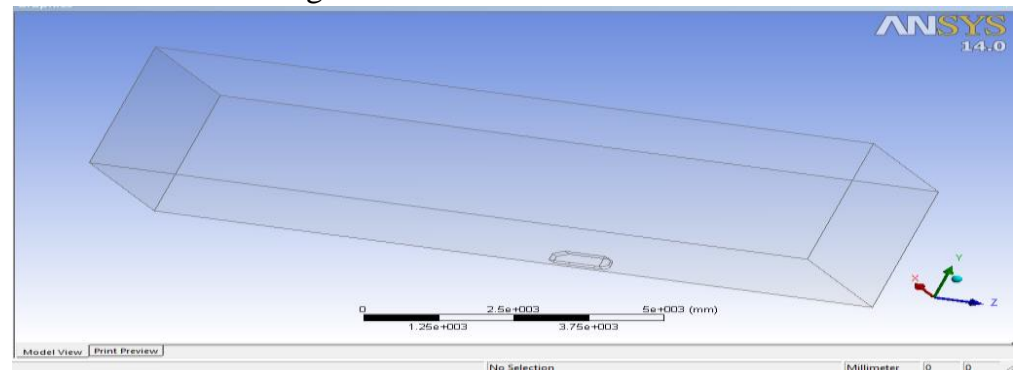

Fig. 9 - Air enclosure in design modeller

\subsection{Meshing}

The name "Finite Element Method" is meant to suggest the technique we apply to all problems. It means that looking at the geometry, the shape of a region, and immediately imagines it broken down into smaller sub-regions. The idea is that we are going to use a simple approximation method, but the errors in this approximation method become unnoticeable as the size of the sub-region gets smaller. So if we use enough small sub-regions, approximated over each one, and then stitch all the answers back together, we can get as smooth and realizable an answer to our original full size problem, as we want. Meshing was carried out in ANSYS meshing tool and the following specifications were used: 
Relevance center: coarse, smoothing: high, Transition: slow, Initisal size seed: Active assembly, Min. size: $1 \mathrm{~mm}$, Max. Size: 250mm, Advanced Size functions: Proximity and Curvature.

\subsection{Modifications In Meshing}

Element size on Ahmed body walls and rollers are still too large but the size of domain air surrounding is good. So, some modifications on Ahmed body were done for getting better result in our analyses, which are explained as follows:

- $\quad$ Roller Sizing- The size of roller meshing is limited to $2 \mathrm{~mm}$.

- Face Sizing- The size of faces meshing is limited to $10 \mathrm{~mm}$.

\subsubsection{Inflation Layer}

Often, the velocity and temperature gradients normal to a wall is typically much larger than the gradients parallel to the wall. The inflation layer controlis designed to create thin elements that can capture the normal gradient with minimal elements; use of inflation layers is done to correctly capture the velocity and temperature gradients near no-slip walls.

Program controlled inflation (as shown in fig-10, 11) with First aspect ratio of 5, growth rate of $20 \%$ of 5 layers with 10 smoothing iterations was done [12].

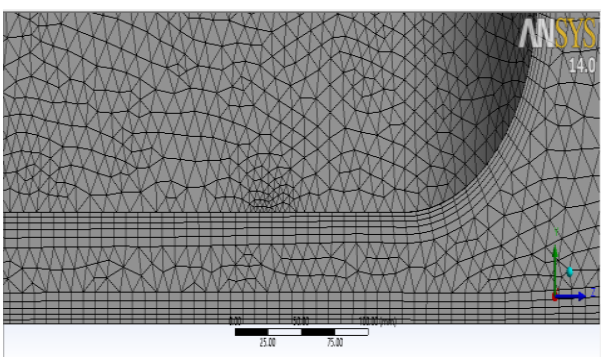

Fig. 10- Prism layer growth front view

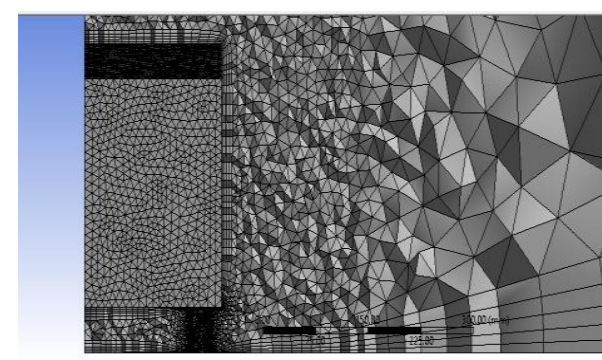

Fig. 11- Prism layer growth sectional view

\subsubsection{Limit Size Boxes}

3 different volumetric boxes (as shown in fig-12) were created around the model so that the meshing size can be limited there, as it gives better control on the result and analysis.

Size of mesh is about $10 \mathrm{~mm}$ at $40 \mathrm{~m} / \mathrm{sec}$.

1. Car Box: A box around the body was created and its sizing was limited to $15 \mathrm{~mm}$.

2. Wake Box: It is done to limit the size at the wake region of the body to $10 \mathrm{~mm}$ to capture the wake velocity contours more accurately.

3. Under box: It is done to limit the size of mesh under the body to $10 \mathrm{~mm}$.

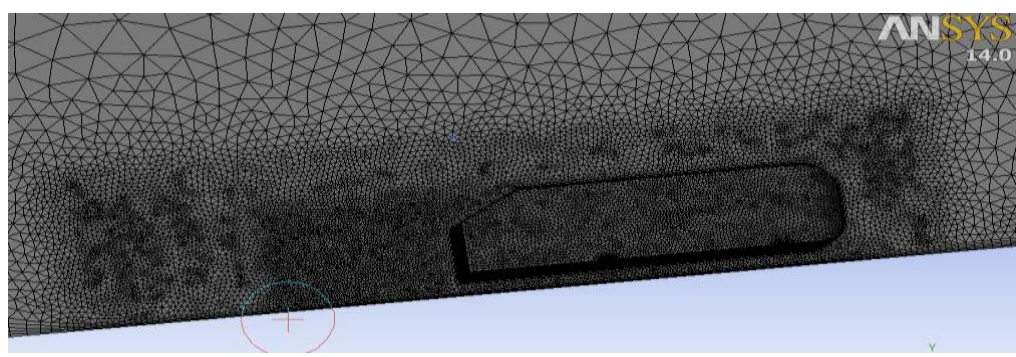

Fig. 12- Meshing of our body

\subsection{Steady Flow Numerical Simulation}

Computational resources for the steady flow numerical simulations are done on Dell work station (1 CPU $1.8 \mathrm{GHz}$ and 8GB RAM) and Windows 8 OS. Realizable k-epsilon model non-equilibrium wall function is used with Inlet velocity V $=40 \mathrm{~m} / \mathrm{s}$, turbulence intensity at inlet is about $1 \%$ and turbulence viscosity ratio is 10 at inlet,turbulence intensity is about $5 \%$ and turbulence viscosity ratio is 10 at outlet. Density of air is 1.225 $\mathrm{kg} / \mathrm{m}^{3}$, temperature is $288.16 \mathrm{~K}$ and viscosity is $1.7894 \mathrm{e}^{-05} \mathrm{~kg} / \mathrm{m}^{4}$. Boundary conditions are - uniform velocity at inlet, uniform pressure at outlet, symmetry at lateral and at top wall of the model and moving wall at floor. 
Solution method utilizes pressure-velocity coupling scheme as coupled with gradient: least square cell based method, pressure as standard and with momentum. Turbulence kinetic energy, turbulence dissipation rate as first order upwind for the initial 100 iterations and correspondingly as second order upwind for the next 500 iterations. Moreover, turbulence viscosity factor is taken as 0.8 for the initial 100 iterations and consequently taken as 0.95 for the remaining 500 iterations.

Analysis of numerous configurations of multifarious modifications of the benchmark model of Ahmed body with 10 degree rear slant angle iscarried out on ANSYS Workbench. Roof and Side cambering is done at 5 varied $\mathrm{H} / \mathrm{L}$ ratioseach, ranging from 0.02 to 1.00 with an increment of 0.02 where $\mathrm{H}$ is the height of camber and $\mathrm{L}$ is the lengthsubjected to cambering. Underbody rear end upward taper is implemented at 4 different taper angles ranging from 5 to 20 degree with an increment of 5 degree. Rear side panel taper is carried out on 3 different lengths of taper of 50,100\&150mm. Rear end tail extension are carried out on 3 extensions of $180,360 \& 540 \mathrm{~mm}$. Chamfered nose is done at $100 \mathrm{~mm}$ nose length. Therefore, a cumulative total of 22 analyses have been carried out including that of benchmark model of Ahmed body.

\subsection{Pressure Distribution}

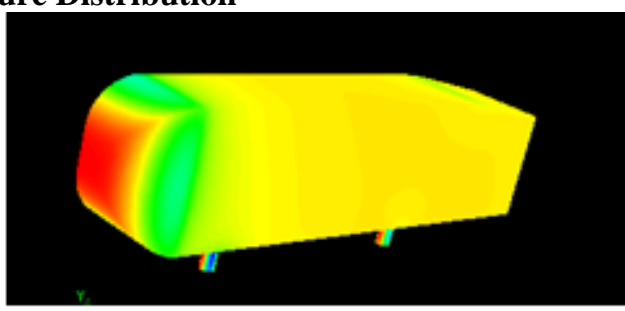

(a)

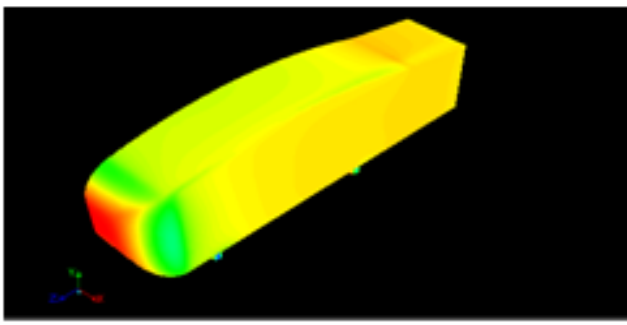

(c)

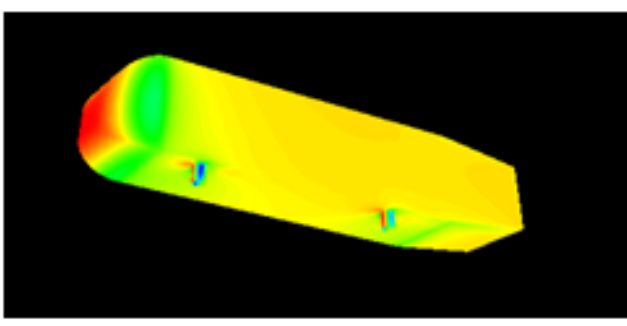

(e)

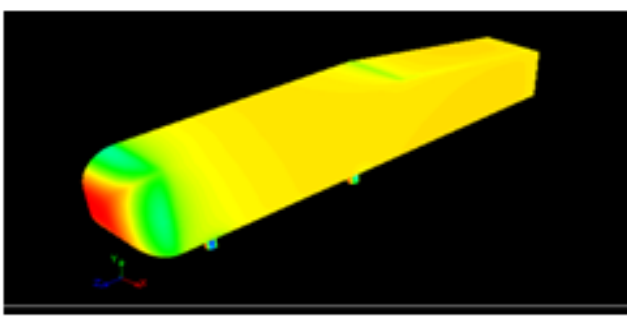

(g)

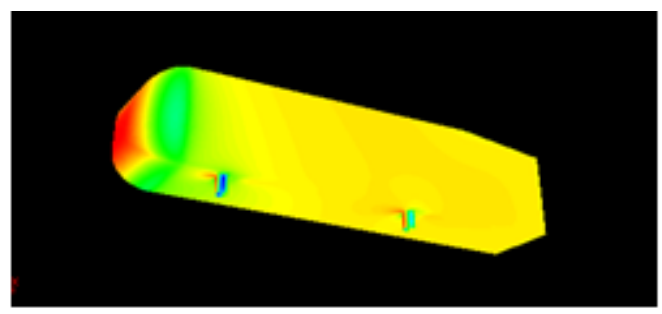

(b)

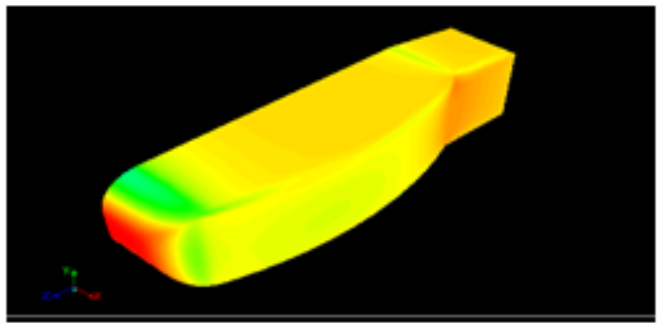

(d)

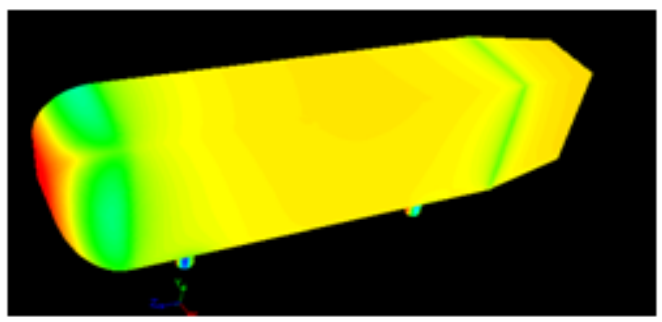

(f)

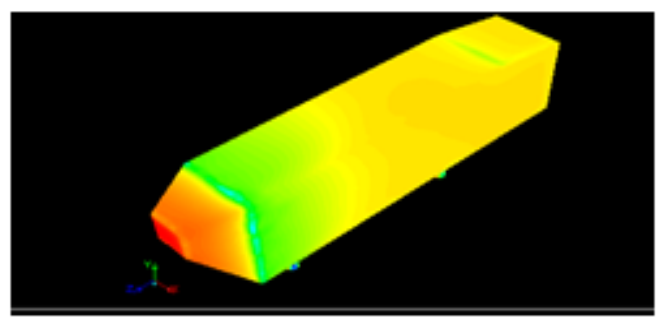

(h)

Fig. 13- Pressure distribution: (a) Benchmark model (Frontal view), (b) Benchmark model (Underbody view), (c) Roof panel cambering, (d) Side panel cambering, (e) Underbody read end upward taper, (f) Rear side panel taper, (g) Rear end tail extension, (h) Frontal area chamfered, 
The pressure distribution gives us the pressure of air over the various surfaces on the geometry of the model. The red, yellow and green areas depict regions of high, medium and low pressure respectively. The air becomes almost stagnant as it strikes the vehicle which results in air exerting very high pressure on front engine grill of the vehicle represented by the red area. The airflow then gets divided between the upper and lower surface of the vehicle. The higher pressure air on front surface accelerates as it travels over the curved nose surface of Ahmed body, causing the pressure to drop. This lower pressure creates lifts over the roof surface as the air passes over it. As the air continues to flow and make its way to the rear, a notch is created by the rear slant owing to flow separation, leaving a vacuum or low pressure space which the air is not able to fill properly. The resulting lower pressure creates lift which then acts upon the surface of the rear slant.

For the multifarious modifications, the pressure distribution is explained as follows:

- Benchmark model ((Frontal \& Underbody view)- As shown in fig-13(a)\&(b), the pressure distribution of the benchmark model features high pressure (red) region on the frontal surface, followed by transition to a narrow region of low pressure (green) around the edges of the frontal surface which finally merges into medium pressure (yellow) region which that encompasses the entire surface of the roof, underbody and side panels.

- Roof panel cambering- As shown in fig-13(c), it is observed that the pressure on roof panel decreases as compared to that of the benchmark model on the overall surface of camber with the surface turning comparatively green (from yellow), indicating lower pressure with respect to the benchmark model.

- Side panel cambering- A similar trend is observed with pressure on side panels being lower(even than that on the roof) on the overall surface of camber than that on theside panels of the benchmark model, as exhibited in fig-13(d).

- Underbody read end upward taper-The rear end upward taper model has lower pressure on the onsetoftaper and at the rear end, functioning to reduce the drag at the through pressure recovery, owing to diffuser-like action as is evident in fig-13(e).

- Rear side panel taper-For the case of rear side panel taper, there is slight decrease in pressure at the onset of taper of side panelsas is apparent in fig-13(f), and consequently, at the rear end.

- Rear end tail extension-There is no marked change in pressure distribution of rear tail extension model as shown in fig- $13(\mathrm{~g})$, because the modification is in the form of continuation of existing geometry and no alteration in contour.

- Frontal area chamfered-High pressure acts on the entirety of the nose of the Ahmed body as evident by the expanse of red color on theentire frontal surface, as exhibited in fig-13(h).

\subsection{Velocity Contours}

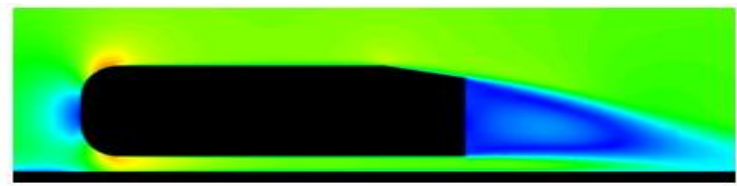

(a)

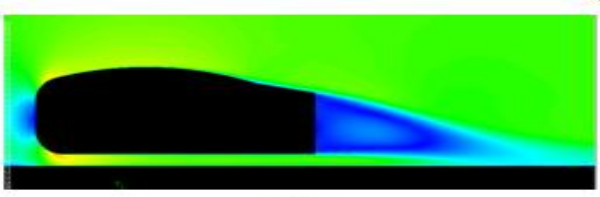

(b)

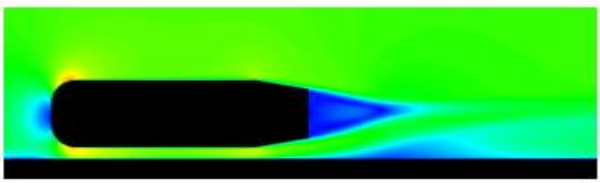

(d)

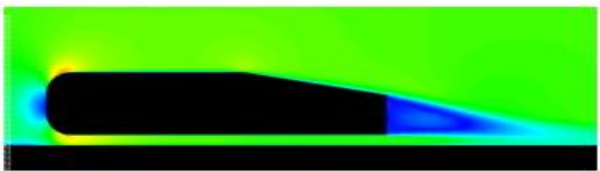

(f)

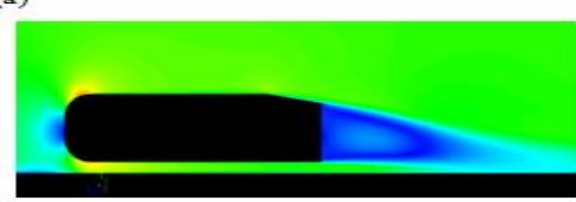

(c)

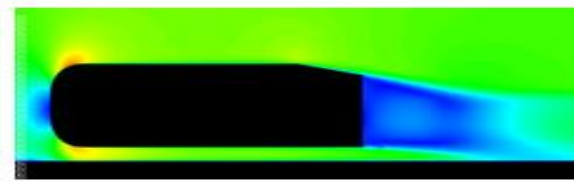

(e)

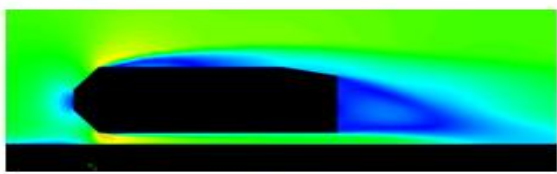

(g)

Fig. 14- Velocity contours: (a) Benchmark model (Side view), (b) Roof panel cambering, (c) Side panel cambering, (d) Underbody read end upward taper, (e) Rear side panel taper,

(f) Rear end tail extension, (g) Frontal area chamfered 
Velocity contours gives us the velocity of air along different geometry sections of the Ahmed body. The green area corresponds to the velocity $40 \mathrm{~m} / \mathrm{sec}$, while blue area shows low velocity region and red, yellow area gives high velocity region. The velocity contours extending behind the body indicate the low velocity wake region, which causes the form drag. As the wake area is reduced, the drag-causing eddies (vortices), generated due to the pressure difference between the top and bottom of the Ahmed body at the rear trailing end, come closer together. This result in less kinetic energy being dissipated due to these smaller eddies and consequently less compensation power required by the engine to propel the vehicle forward. It additionally results in higher pressure being applied at the rear surface of the body and consequently less form drag.

For the multifarious modifications, the velocity contours are explained as follows:

- Benchmark model- The benchmark model is characterized by a wide tail of low velocity at the rear originating from the trailing edge of the slant - wake region, as can be observed in fig-14(a). Also, low velocity air at the center of the frontal surface, and high velocity of air at the edges of the frontal surface is witnessed in this case.

- Roof panel cambering- In case of roof panel cambering, a lower velocity is exhibited at the onset of camber on the roofas shown in fig-14(b), and no marked departure in the wake region is observed from that of the benchmark model.

- Side panel cambering- For the side cambering model, a similar trend is observed in the wake region but with the difference of lower velocity occurring along the side panels but remaining the same along the roof as in case of the benchmark model, being evident in fig-14(c).

- Underbody read end upward taper-The rear end upward taper has a comparatively narrow (pointed) wake area at the rear as a result of comparatively higher velocity on the underbody, owing to the geometrical modification, as exhibited in fig-14(d).

- Rear side panel taper-For the case of rear side panel taper, there is a similar reduction in wake area but this time along its width accounting for the wake region ending prematurely along the length than that in case of benchmark model, ascan be observed in fig-14(e).

- Rear end tail extension-There is a decrease in rear height for the case of rear tail extension due to modification in the geometry, resulting in decrease of rear wake area as can be seen in fig-14(f).

- Frontal area chamfered- Finally, for the chamfered nose model, low velocity due to boundary layer separation at the edge of the nose on frontal surface of the Ahmed body, continuing throughout the length on the roof of the body and also significantly large wake area is evident in fig-14(g).

\section{Results}

The analyses of the 22 multifarious models carried out yield optimum configurations for each geometrical modification of the benchmark model - Ahmed body having 10 degree rear slant angle. The values of the aerodynamic parameters obtained for the benchmark model are- Coefficient of drag $\left(\mathrm{C}_{\mathrm{d}}\right)=0.238$ and the coefficient of lift $\left(\mathrm{C}_{1}\right)=0.0848$ [2]. For all the configurations for a particular modification (obtained by varying the associated geometrical parameter), the drag and lift coefficients are plotted and examined to determine the optimum configuration resulting in least drag and lift coefficients. Furthermore, the underlying root causes are also analysed, comprehended and listed.

\subsection{Roof Camber}

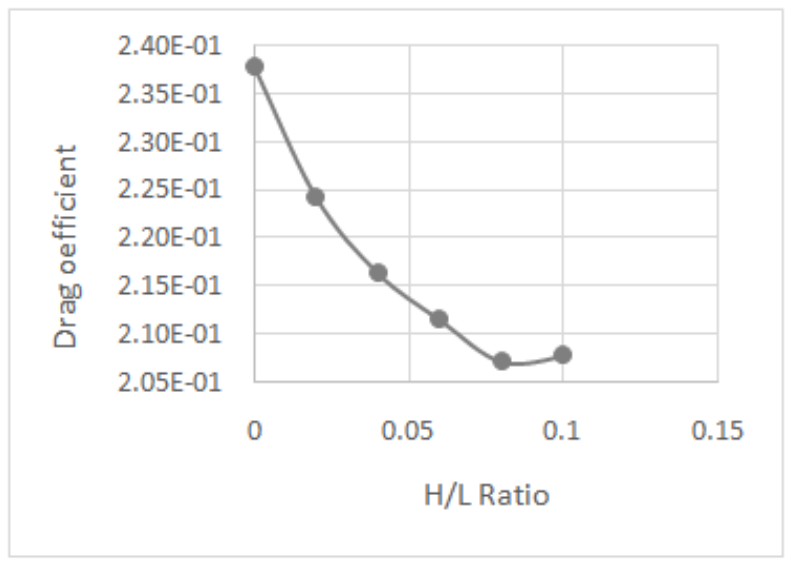

Fig. 15- Roof camber: Drag vs. H/L ratio

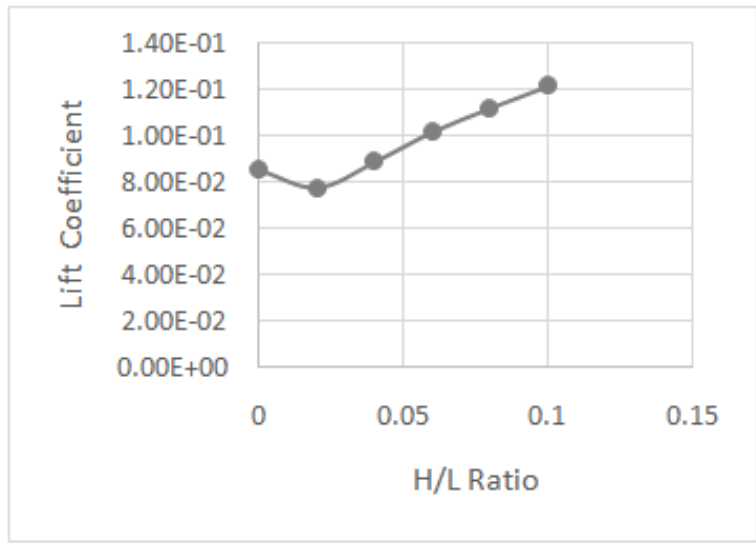

Fig. 16- Roof camber: Lift vs. H/L ratio 
- Roof camber having H/L ratio of 0.08 gives least drag configuration (as shown in fig-15) for this modification due to delayed boundary layer separation and thereafter, drag starts to increase again. This increase is due to increase of roof camber at the expense of enlarging the frontal area and this turns out to be counter-productive.

- Roof camber having H/L ratio of 0.02 gives least lift configuration (as shown in fig-16) for this modification and thereafter, it starts to increase continually due to reduction in pressure over the round curvature of roof.

\subsection{Side Camber}

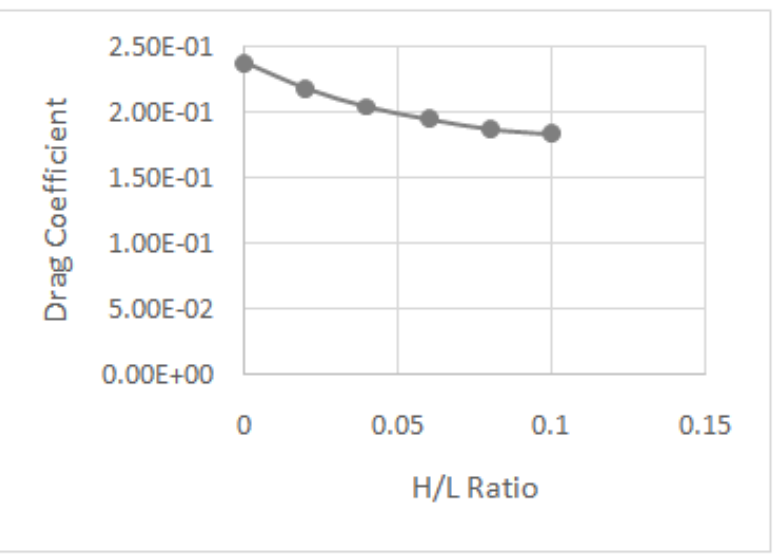

Fig. 17- Side camber: Drag vs. H/L ratio

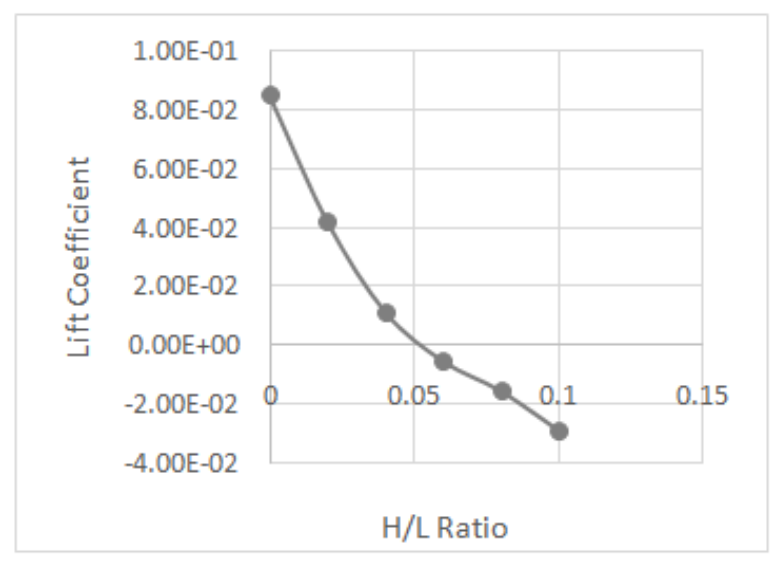

Fig. 18- Side camber: Lift vs. H/L ratio

- Drag coefficient is decreasing with increase in H/L ratio (as exhibited in fig-17) due to delayed boundary layer separation. However, with even further increase in $\mathrm{H} / \mathrm{L}$ ratio, camber increase occurs at the expense of enlarging the frontal area and this turns out to be counter-productive, same as in the case of roof camber.

- Lift coefficient keeps on continually decreasing with increase in H/L ratio (as exhibited in in fig-18) due to reduction in pressure over the round curvature on the side panels of the Ahmed Body resulting in generation of downforce.

\section{3underbody Rear End Upward Taper}

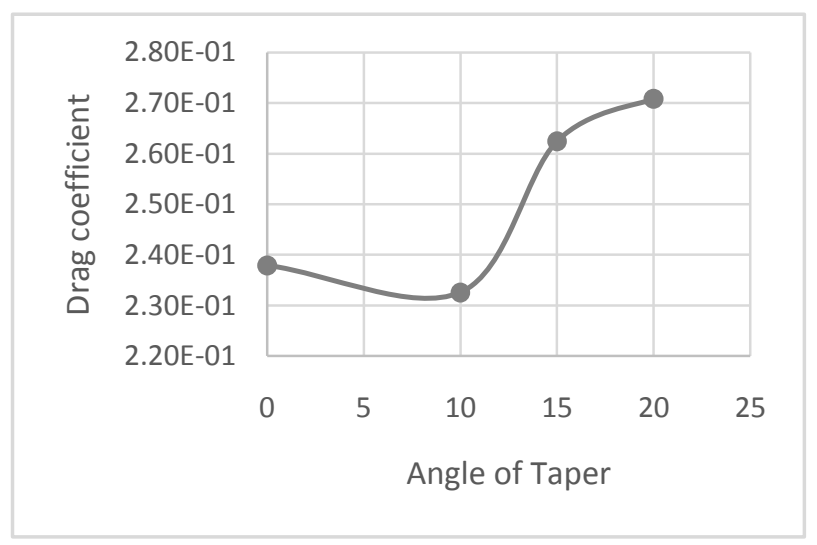

Fig. 19- Underbody Rear End Upward Taper: Drag vs. Angle

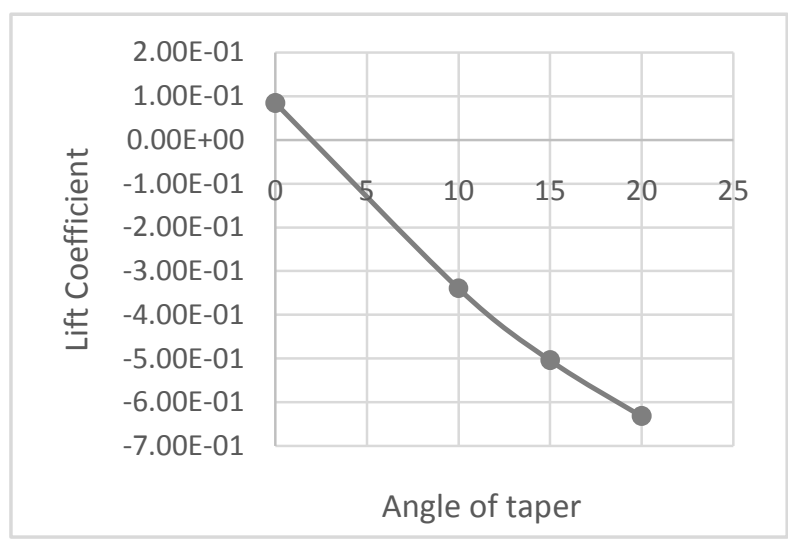

Fig. 20- Underbody Rear End Upward Taper: Lift vs. angle

- Least Drag coefficient obtained at angle of 10 degree (as portrayed in fig-19) for the rear end upward taper due to the smaller wake region formed. However, with further increase in angle, drag coefficient increases as it give rise to boundary layer separation at steep inclined rear underbody taper panel.

- Lift is continually decreasing with increase in angle of taper (as portrayed in fig-20) due to the increase in velocity under the body at rear end which give rise to low pressure formation, consequently resulting generation of downward force. 


\subsection{Rear Side Panel Taper}

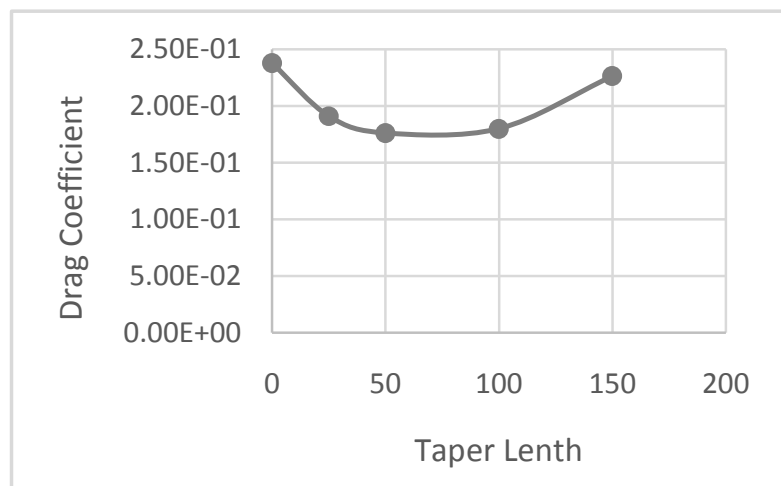

Fig. 21- Rear Side Panel: Drag vs. Taper length

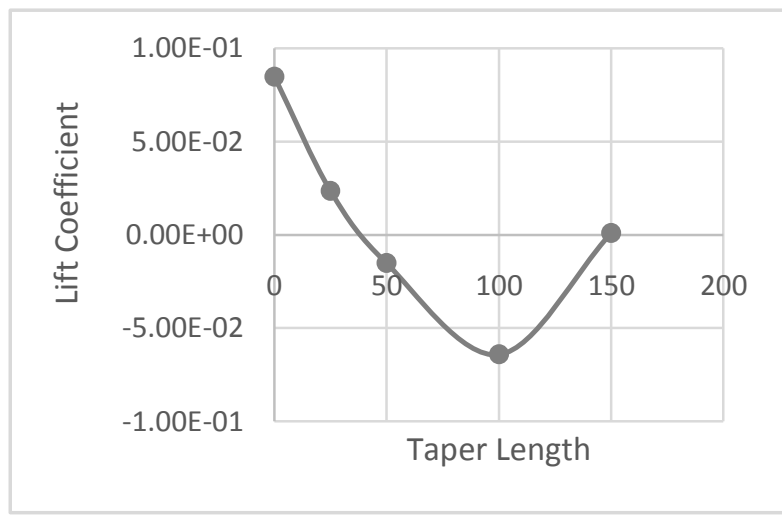

Fig. 22- Rear Side Panel: Lift vs. Taper length

- With rear side panel taper till $50 \mathrm{~mm}$, a marked reduction in drag coefficient is observed (as is evident in fig-21) due to reduction in wake area at the rear end. There is negligible change between taper of 50 $100 \mathrm{~mm}$, and thereafter it starts to increase significantly due to flow separation owing to steep incline of rear panel taper.

- Rear side panel of $100 \mathrm{~mm}$ gives least lift configuration for this modification (as is evident in fig-22) and thereafter, it starts to increase.

\subsection{Rear End Tail Extension}

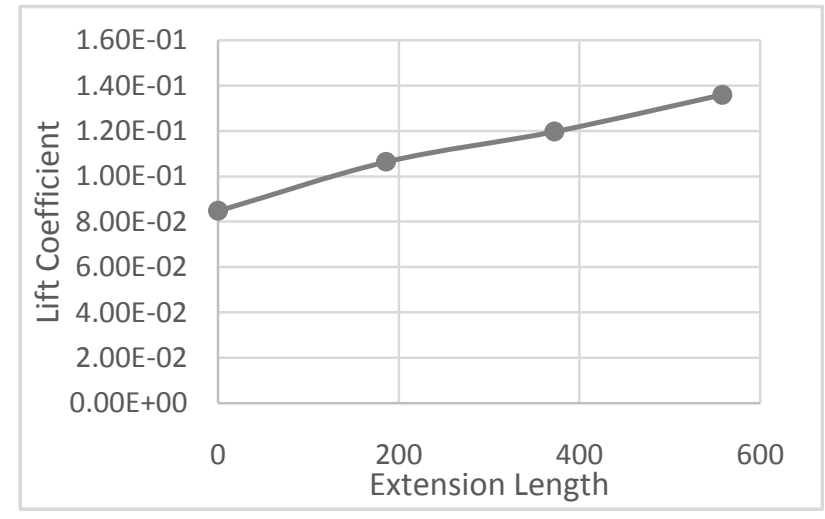

Fig. 23- Rear End Tail Extension: Drag vs. Extension length

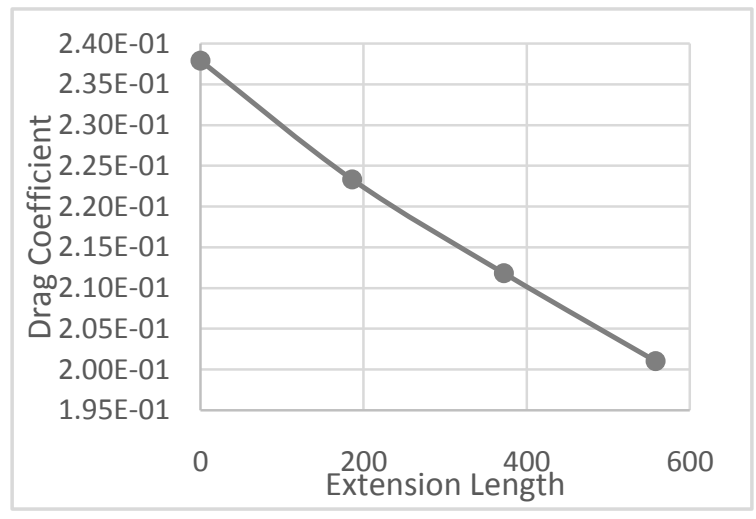

Fig. 24- Rear End Tail Extension: Lift vs. Extension length

- Drag coefficient is decreasing with increasing rear extension (as seen in fig-23) due to reduction in wake region but this shape is practically inconvenient and impractical from design considerations. Conversely if the rear end tail is cropped at various lengths and curved downwards there is an increase in drag coefficient with each reduction in tail length beyond the rear wheels.

- Lift is continually increasing with increasing rear extension (as seen in fig-24) due to increase in velocity and consequent reduction in pressure on the roof owing to larger dimension of surface over which the area flows. 


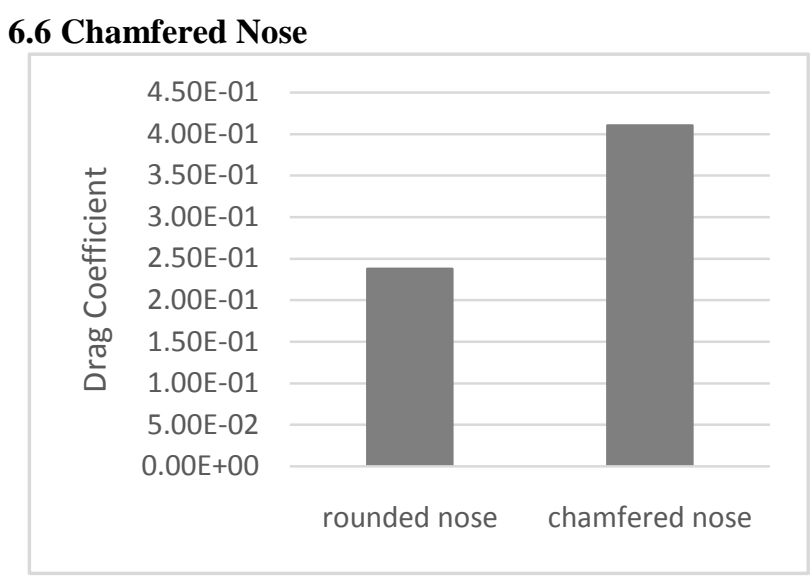

Fig. 25- Chamfered vs. Rounded nose (drag)

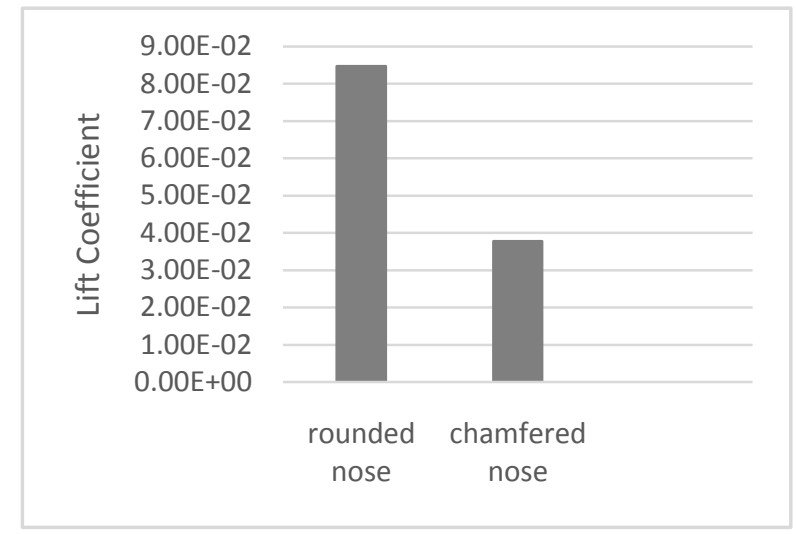

Fig. 26- Chamfered vs. Rounded nose (lift)

- Drag coefficient is increasing in chamfered nose model, as shown in fig-25, as compared to rounded nose (benchmark) model due to boundary layer separation occurring at the sharp edges.

- Lift is decreasing in chamfered nose model as compared to rounded nose(benchmark) model, as shown in fig-26, due to flow of air over the roof at the high velocity which results in low pressure and generation of upward force.

\section{Conclusion}

CFD results for multifarious variants of modifications of Ahmed Body with rear slant angle 10 degree are presented after carrying out their design, development and subsequent analysis. Thereafter, comparison and examination of variants of a particular modification is done to establish the effectiveness of each variant. The following conclusions can be drawn from the results:

1. Coefficient of drag $\left(C_{d}\right)$ of benchmark model - Ahmed Body with 10 degree rear slant angle - is obtained as 0.238 and the coefficient of lift $\left(\mathrm{C}_{1}\right)$ as 0.0848 .

2. The optimum configuration of roof camber modification for least drag is obtained with $\mathbf{H} / \mathbf{L}$ ratio of $\mathbf{0 . 0 8}$ resulting in $C_{d}$ of 0.20705 , which implies reduction of $13 \%$ and resulting in $C_{1}$ of 0.112 .

3. The optimum configuration of side camber modification for least drag is obtained with $\mathbf{H} / \mathbf{L}$ ratio of $\mathbf{0 . 1 0}$ resulting in $\mathrm{C}_{\mathrm{d}}$ of 0.184 , which implies drag reduction of $22.7 \%$ and resulting in $C_{1}$ of -0.02904 , which implies lift reduction of $\mathbf{1 3 4 \%}$.

4. The optimum configuration of underbody rear end upward taper modification for least drag is obtained with angle of 10 degree resulting in $C_{d}$ of 0.2326 , which implies drag reduction of $2.27 \%$ and resulting in $C_{1}$ of -0.339 , which implies lift reduction of $499.7 \%$.

5. The optimum configuration of rear side panel taper modification for least drag is obtained with taper of $\mathbf{5 0 m m}$ resulting in $C_{d}$ of 0.1761 , which implies drag reduction of $26 \%$ and resulting in $C_{1}$ of -0.0152 , which implies liftreduction of $\mathbf{1 1 7 . 9 \%}$.

6. The feasible configuration of rear end tail extension for reducing drag is obtained with extension of 186mm resulting in $C_{d}$ of 0.2233 , which implies drag reduction of $6.18 \%$ and resulting in $C_{1}$ of 0.1064 . The drag goes on continually decreasing with increase in extension dimension but, it becomes practically infeasible to apply.

7. The configuration of chamfered nose modification, instead of fillet results in $\mathrm{C}_{\mathrm{d}}$ of 0.4102 , which implies drag increase of $72 \%$ and resulting in $C_{1}$ of 0.03781 .

Therefore, the optimum configurations for least drag and lift coefficients, corresponding to each modification of the geometry of the benchmark model are obtained enhancing the aerodynamic behaviour and consequently, the efficiency of the automobile. Future scope for application of these results include coupling of numerous feasible complementarymodifications together or coupling with suitable active and passive drag reducing devices (add-ons) to further boost the efficiency of the automobile. 


\section{References}

[1] S. R. Ahmed, G. Ramm, G. Faitin, 'Some Salient Features of the Time - Averaged Ground Vehicle Wake', (SAE-TP-840300) Society of Automotive Engineers, Inc., Warrendale, PA, 1984.

[2] Saurabh Banga, Md. Zunaid, Naushad Ahmad Ansari, Sagar Sharma, Rohit Singh Dungriyal, 'CFD Simulation of Flow around External Vehicle: Ahmed Body’DOI: 10.9790/1684-12438794, IOSR Journal of Mechanical and Civil Engineering (IOSR-JMCE).

[3] T. Han, D. C. Hammond, C. J. Sagi, 'Optimization of bluff body for minimum Drag in ground proximity', AIAA Journal, Vol. 30, No. 4, pp. 882-889, doi: 10.2514/3.11005, 1992.

[4] G. Pujals, S. Depardon, C. Cossu, 'Drag reduction of a 3D bluff body using coherent stream wise streaks', Springer, Exp Fluids (2010) 49:1085-1094, DOI 10.1007/s00348-010-0857-5, 2010.

[5] Robert J. Englar, 'Advanced Aerodynamic Devices to Improve the Performance, Economics, Handling and Safety of Heavy Vehicles', SAE 2001-01-2072, Georgia Tech Research Institute Aerospace Transportation \& Advanced Systems Lab, 2001.

[6] Shubham Singh, M. Zunaid, Naushad Ahmad Ansari, Shikha Bahirani, Sumit Dhall, Sandeep Kumar, 'Numerical Study of the Generic Sports Utility Vehicle Design with a Drag Reduction Add-On Device', Hindawi Publishing Corporation Journal of Computational Engineering Article ID 785294, Volume 2014.

[7] A. Lamond, J. J. Kennedy, M. T. Stickland, 'An investigation into unsteady base bleed for drag reduction in bluff two box SUV's', Proceedings of the 4th European Automotive Simulation Conference (EASC'09), Munich, Germany, 2009.

[8] K. Bahram, S. Zhang, C. Koromilas, 'Experimental and computational study of unsteady wake flow behind a bluff body with a drag reduction device', SAE2001-01B-207, 2001

[9] M. H. Coanda, "Propelling device," French Patent no. 796843, 1936.

[10] E.M. Wahba., H. Al-Marzooqi, M. Shaath, M. Shahin, T. El-Dhmashawy, 'Aerodynamic Drag Reduction for Ground Vehicles using Lateral Guide Vanes', CFD Letters, vol.4, no.2, pp.68-79, 2012.

[11] T. Gustavsson and T. Melin, 'Application of Vortex generators to a blunt body', Technical Report KTH, Department of Aeronautical and Vehicle Engineering, Royal Institute of Technology, Stockholm,Sweden,2006.

[12] Marco Lanfrit, 'Best practice guidelines for handlingAutomotive External Aerodynamics with FLUENT', Version 1.2 (Feb 9th 2005), Fluent Deutschland Gmbh Birkenweg 14a 64295 Darmstadt/Germany. 